Sustained spheromak coaxial gun operation in the presence of an $n=1$ magnetic distortion

C. T. Holcomb, T. R. Jarboe, D. N. Hill, S. Woodruff, R. D. Wood

September 13, 2005

Physics of Plasmas 
This document was prepared as an account of work sponsored by an agency of the United States Government. Neither the United States Government nor the University of California nor any of their employees, makes any warranty, express or implied, or assumes any legal liability or responsibility for the accuracy, completeness, or usefulness of any information, apparatus, product, or process disclosed, or represents that its use would not infringe privately owned rights. Reference herein to any specific commercial product, process, or service by trade name, trademark, manufacturer, or otherwise, does not necessarily constitute or imply its endorsement, recommendation, or favoring by the United States Government or the University of California. The views and opinions of authors expressed herein do not necessarily state or reflect those of the United States Government or the University of California, and shall not be used for advertising or product endorsement purposes. 


\title{
Sustained spheromak coaxial gun operation in the presence of an $n=1$ magnetic distortion
}

C.T. Holcomb

Lawrence Livermore National Laboratory, Livermore, California 94550

T.R. Jarboe

Aerospace and Energetics Research Program, University of Washington, Seattle, Washington 98195-2250

D.N. Hill, S. Woodruff, R.D. Wood

Lawrence Livermore National Laboratory, Livermore, California 94550

\begin{abstract}
The Sustained Spheromak Physics Experiment (SSPX) uses a magnetized coaxial gun to form and sustain spheromaks by helicity injection. Internal probes give the magnetic profile within the gun. Analysis of these data show that a number of commonly applied assumptions are not completely correct, and some previously unrecognized processes may be at work. Specifically, the fraction of the available vacuum flux spanning the gun that is stretched out of the gun is variable and not usually $100 \%$. The $n=1$ mode that is present during sustained discharges has its largest value of $\delta \mathrm{B} / \mathrm{B}$ within the gun, so that instantaneously B within the gun is not axisymmetric. By applying a rigid-rotor model to account for the mode, the instantaneous field and current structure within the gun are determined. The current density is also highly non-axisymmetric and the local value of $\lambda \equiv \mu_{0} \mathrm{j}_{\|} / \mathrm{B}$ is not constant, although the global value $\lambda_{\mathrm{g}} \equiv \mu_{0} \mathrm{I}_{\mathrm{g}} / \psi_{\mathrm{g}}$ closely matches that expected by axisymmetric models. The current distribution near the gun muzzle suggests cross-field current exists, and this is explained as a line-tying reaction to plasma rotation.
\end{abstract}

PACS: 52.55.Hc, 52.35.Py, 52.30.Cv 


\section{INTRODUCTION}

A spheromak ${ }^{1}$ is a simply-connected toroidal magnetic equilibrium that approaches a force-free state described by $\nabla \times \mathbf{B}=\lambda \mathbf{B}$. The eigenvalue $\lambda \equiv \mu_{0} \mathrm{j}_{\|} / \mathrm{B}$ is a flux surface quantity that approaches a global constant via relaxation while conserving magnetic helicity. The helicity $K \equiv \int \mathbf{A} \cdot \mathbf{B} d V$, where $\mathbf{A}$ is the magnetic vector potential, is a measure of flux linkage within the spheromak volume. This volume is usually defined by a conducting wall referred to as 'the flux conserver'. Helicity is conserved on a timescale long compared to the Alfven and reconnection times, and only decays on the resistive diffusion timescale. ${ }^{2}$ This implies that a spheromak equilibrium can be sustained by injecting helicity at a rate greater than or equal to the helicity decay rate. ${ }^{3}$ One way this is accomplished is to connect a magnetized coaxial gun to the flux conserver. The resulting helicity balance equation is $d K / d t=2 V_{g} \psi_{g}-K / \tau_{K}$, where $V_{g}$ is the gun voltage across the gun flux $\psi_{g}$ that penetrates both electrodes, and $\tau_{K}$ is the helicity decay time. This equation describes the helicity in the entire flux conserving volume that contains plasma capable of carrying current, which includes the flux conserver and the coaxial gun up to the insulating break.

The helicity balance description may be used to predict the final equilibrium state brought about by relaxation. However, it does not predict the relaxation mechanism(s) that will accomplish this, i.e. it does not describe how current initially driven along open flux at the edge leads to current driven on closed flux within the core on a timescale shorter than $\tau_{K}$. Various magnetic dynamo ${ }^{4}$ mechanisms are thought to be responsible, and all rely on a time-varying fluctuation of the magnetic field. 
During sustained spheromak experiments, magnetic probes at the edge almost universally observe a time-varying departure from toroidal symmetry of the form $\mathrm{e}^{\mathrm{i}(\omega t+n \phi)}$ with $n=1$. The toroidal angle is $\phi$ and $\omega$ is referred to as 'the $n=1$ frequency'. The Spheromak Experiment (SPHEX) ${ }^{5,6}$ made internal floating potential, magnetic probe, and Rogowksi (current) probe measurements all the way to the symmetry axis of the equatorial plane of a spheromak while this " $\mathrm{n}=1$ mode" was active. The $\mathrm{n}=1$ mode was identified as a kink instability of the open flux tube aligned with the geometric axis. The displaced open-flux tube axis rotated about the geometric axis at the $\mathrm{n}=1$ frequency, as did a large local distortion of open flux near the wall. (See Figure 9 in Ref. 5). In general, the $\mathrm{n}=1$ mode dominates the spheromak during sustainement, and is believed to be responsible for relaxation current drive. ${ }^{7,8}$

Historically, less attention has been given to the field and current structure within the coaxial gun that injects helicity and sustains the spheromak than to the spheromak equilibrium itself. Three assumptions are commonly applied to the gun that simplify analysis of coaxial helicity injection: 1 . The field and current are axisymmetric to first order $^{1}$; 2. Relaxation on the open flux is rapid (i.e. $\tau_{\text {relax }}<<\tau_{\text {resistive }}$ ) so that the current density distribution on the electrodes and open flux is uniform, parallel to the field, and $\lambda$ on the open flux is spatially constant ${ }^{9}$; and 3 . After formation, $100 \%$ of $\psi_{\mathrm{g}}$ remains connected to the electrodes while linking the magnetic axis of the spheromak, thus contributing to the helicity injected into the spheromak separatrix during sustainment. ${ }^{3}$

This paper discusses new measurements and analysis of the Sustained Spheromak Physics Experiment (SSPX) that show significant variation from these three assumptions. Internal magnetic measurements inside the coaxial gun near its connection to the main 
flux conserver show that during sustainment with an $\mathrm{n}=1$ mode present, the open field fluctuation (peak-to-peak) $\delta B_{z} / B_{z}=15-20 \%$ across the entire gun width. Since the frequency of the $\mathrm{n}=1$ fluctuation is relatively constant and does not vary with radius a rigid-rotor model is applied to the data to obtain a "snap-shot" of the instantaneous asymmetric field and current distribution in the gun. For identical shot configurations, the total flux stretched out of the gun can fall within the range $0.65 \psi_{\mathrm{g}}$ to $\psi_{\mathrm{g}}$, with shot-to-shot and time-averages of $0.80 \psi_{\mathrm{g}}$, meaning that some fraction of $\psi_{\mathrm{g}}$ does not link the spheromak formed in the flux conserver. The local value of $\lambda$ on the open flux is not uniform, and one implication of this is the existence of current perpendicular to the field. Finally, this cross-field current is shown to put a force on the plasma in the toroidal direction, and this is interpreted as a line-tying reaction to plasma rotation that is observed in spheromaks.

\section{EXPERIMENTAL SET-UP}

The SSPX goals ${ }^{9,10}$, parameters ${ }^{11}$ and diagnostics ${ }^{12}$ have been described in detail elsewhere. Fig. 1 shows the SSPX cross section and labels the features that will be referred to in this paper, including the coaxial gun, flux conserver, gun flux, and one of three magnetic probe arrays used in this study. This study is based on an operating configuration that uses only the central gun coil to produce $\psi_{\mathrm{g}}=18 \mathrm{mWb}$, and a $1.5 \mathrm{MJ}$ pulse forming sustainment bank connected to the gun that drives current for about 4 ms. During the middle $2 \mathrm{~ms}$ of each shot, helicity balance estimates ${ }^{13}$ predict a net increase in helicity and the self-generated equilibrium field is observed to be roughly constant or growing slowly. A coherent $n=1$ mode is visible on all magnetic signals during this time (see Figure 2). 
The internal magnetic probe arrays are mounted on linear actuators to allow complete retraction behind the outer electrode wall. Two arrays are at $\phi=292^{\circ}$ (the one shown in Figure 1 and another $10 \mathrm{~cm}$ above it) and a third array is at $\phi=90^{\circ}$ at the same height as the array shown in Figure 1. Each array has six radial, six axial, and six toroidal field probes to span the gun gap. When fully inserted, the closest probe set is $10 \mathrm{~mm}$ away from the surface of the inner electrode. Consecutive discharges with identical settings are compared with and without probes inserted to gauge the perturbation. This shot configuration shows no discernable difference on edge magnetics, gun voltage, or peak electron temperature $(40-50 \mathrm{eV})$ with or without probes inserted, suggesting that perturbation is negligible.

\section{DATA ANALYSIS AND RESULTS}

Figure 2 shows three internal $B_{z}$ probe signals on the array shown in Figure 1 while a coherent and steady $25 \mathrm{kHz} n=1$ mode is present. The $\delta B_{z} / B_{z} \approx 15-20 \%$ across the gun gap, so an analysis is required that takes into account the $n=1$ asymmetry. All internal probes show $n=1$ fluctuations of similar amplitude and the frequency does not change with radius. This suggests the rigid rotation of a structure that evolves on a timescale longer than the rotation period.

The SPHEX group made similar observations with various internal probes at the spheromak midplane. It was assumed that all signals having strong fluctuations at the $n=1$ frequency were caused by the rotation of a structure about the geometric axis, thus allowing time variations to be interpreted as spatial variations in azimuth. A similar rigidrotor analysis is preformed with data from the internal probes in the SSPX gun. First the 
vacuum field value at each probe position is added to each integrated B-dot signal to give absolute magnetic field. For each field component, measurements in the gun and the edge are interpolated to get a radial profile at each time. An example is shown in Figure 3. With a $3 \mathrm{MHz}$ digitizer a typical $25 \mathrm{kHz}$ mode frequency gives about 120 sampled times per mode period. Using $d \phi=\omega d t$ this is equivalent to a toroidal grid spacing of 3 degrees, so the entire annular area of the gun gap is sampled by a cylindrical grid containing about 2000 points. Color-filled contour plots of $\mathrm{B}_{\mathrm{Z}}, \mathrm{B}_{\mathrm{T}}$, and $\mathrm{B}_{\mathrm{r}}$ between the electrodes are shown in Fig. 4 with the mouth of the gun and the flux conserver into the page. Negative values of $B_{Z}$ (blue) point into the page and the mode rotates clockwise. The contours show the flux distorts into an asymmetric concentration within the gap.

The total axial flux passing through this annulus in either direction is found by integration of the negative or positive $\mathrm{B}_{\mathrm{z}}$ over the area using a 2-D trapezoid rule. The flux directed out of (in to) the gun at the probe array is added to the known vacuum flux penetrating the outer (inner) electrode below the height of the array. The resulting quantities are equal within error and equal to the total vacuum flux ejected from the gun. This quantity is expected to be identical to $\psi_{\mathrm{g}}$, but the measurements show otherwise. For identical discharge conditions, the injected flux is as high as $100 \%$ of $\psi_{\mathrm{g}}$ and as low as $65 \%$, with an uncertainty of $6 \%$. The average value over time and many shots is $80 \%$, which roughly corresponds to the fraction of $\psi_{\mathrm{g}}$ anchored to the outer wall at and below the gas valves. Figure 5 shows the ejected flux versus time for two identical discharge settings. Values greater than $100 \%$ may be the result of closed equilibrium flux penetrating the gun gap and the probe array. 
The axial current density is $\left(\operatorname{curl} \mathbf{B} / \mu_{0}\right)_{\mathrm{z}}$. This is calculated using the probe measurements and the rigid rotor model for variation in $\phi$. If the plasma is force free, then the local $\lambda$ is $\mu_{0} \mathrm{j}_{z} / \mathrm{B}_{\mathrm{z}}$ at all points in the annulus, and this is shown in Figure 6 for the same shot and times as Figure 4. Clearly $\lambda$ is not constant in the gun. Values with an uncertainty greater than $2 \lambda$ have been marked and should be discounted (these are mostly in locations where $B_{z}$ and $j_{z}$ are small). Even after eliminating points with large error, the local $\lambda$ still departs considerably from the symmetric ejection threshold prediction $\pi / \Delta=20 \mathrm{~m}^{-1}$ but the spatially-averaged value is usually in the range of 10 to $20 \mathrm{~m}^{-1}$ and approximately equal to the global $\lambda_{\mathrm{g}} \equiv \mu_{0} \mathrm{I}_{\mathrm{g}} / \psi_{\mathrm{g}}$. For comparison, $\lambda_{\mathrm{g}}=15 \mathrm{~m}^{-1}$ for the shot and time shown in Figure 6.

The axial plasma currents flowing into and out of the gun are found by integrating the positive and negative $j_{z}$ over the annular area. Currents flowing within the inner and outer electrodes are determined by integration of $\mathrm{B}_{\phi}(\mathrm{r}, \phi)$ around each electrode circumference. Within error, the total current leaving the gun is $\mathrm{I}_{\mathrm{g}}$. Kirchoff's Law demands that the sum of all injected and return currents measured at the array be zero. Summing the currents always gives a nonzero remainder less than $10 \%$ of $\mathrm{I}_{\mathrm{g}}$, so this condition is satisfied within error. Figure 7 shows the distribution of axial current at the height of a probe array for a typical discharge: current flows out of the gun in plasma $\left(\mathrm{I}_{\mathrm{p}}{ }^{\text {out }}\right)$ and within the outer electrode $\left(\mathrm{I}_{\mathrm{e}}{ }^{\text {out }}\right)$, and it returns to the gun through plasma $\left(\mathrm{I}_{\mathrm{p}}{ }^{\mathrm{in}}\right)$ and within the inner electrode $\left(\mathrm{I}_{\mathrm{e}}{ }^{\text {in }}\right)$. Note that $\mathrm{I}_{\mathrm{p}}{ }^{\text {out }}>\mathrm{I}_{\mathrm{p}}{ }^{\text {in }}$ and $\mathrm{I}_{\mathrm{e}}{ }^{\text {in }}>\mathrm{I}_{\mathrm{e}}{ }^{\text {out }}$ as represented by the arrows in the left hand side of Figure 1. This suggests that some current that initially flows out of the gun along, for example, field line \#1 returns to the gun along a different field line (\#2) that is anchored to the electrodes farther down the gun. 


\section{DISCUSSION}

To summarize, the main results presented here show that the coaxial gun current profile is asymmetric during sustainment with an $n=1$ mode, the fraction of gun flux swept out of the gun is variable for identical shot conditions, and the open-flux current density sharply varies from the simple force-free current equilibrium model.

The variable gun flux utilization suggests that there is a limited penetration of the injected gas into the upper part of the gun before the voltage is applied. The upper part of the gun must be gas starved and plasma-free after breakdown, otherwise current would flow there and stretch all of the gun flux out, but this is not observed. Furthermore we observe the entire gun current at the probe height, which is another indication that no current flows on the flux left in the top of the gun. This poses a difficulty for calculating helicity balance in coaxial gun driven spheromaks: the helicity injection rate is reduced by the presence of a significant fraction of $\psi_{\mathrm{g}}$ that does not support plasma current. (Potential applied to flux without current generates zero helicity). This may only be a problem for SSPX, but it shows the wisdom of experimentally verifying the gun flux utilization in other experiments that use coaxial helicity injection.

The non-uniform local $\lambda$ and unbalanced open field line plasma currents leaving and returning to the gun are quite unexpected. They show that the open field line current is not fully relaxed. The observations in Figure 7 that $\mathrm{I}_{\mathrm{p}}{ }^{\text {out }}>\mathrm{I}_{\mathrm{p}}{ }^{\text {in }}$ and $\mathrm{I}_{\mathrm{e}}{ }^{{ }^{\text {in }}}>\mathrm{I}_{\mathrm{e}}{ }^{\text {out }}$ suggests that the open field line current may not be force-free. To understand why, we must consider the often-misunderstood concept of "line-tying". In an ideal plasma that extends all the way to the flux conserving walls the field lines are "frozen-in" and only move by 
convecting with the fluid. Since the plasma velocity at the flux conserving walls goes to zero by the no-slip boundary condition, field lines that penetrate the wall remain anchored ("line-tied") to the same locations at the walls. To the extent that the plasma is resistive and field lines 'slip' through the plasma, the field lines per unit area penetrating the conducting wall must still remain constant. If a field line anchor point moves along the wall then another field line must move into its previous position at the same rate to maintain constant unit flux in the conducting material. Since the open flux provided by the external coils and the surface area of the walls are finite, field line anchor point wandering could only be allowed in the toroidal direction due to symmetry, and then only if this wandering is perfectly toroidally symmetric to conserve flux. This is highly unlikely, so "line-tying" is a valid principle in general.

Referring again to Figure 1, the poloidal projections of field lines \#1 and \#2 are shown. These are line tied to the walls and the anchor points can not move vertically. Force-free current that starts on field line \#1 at the outer electrode should stay on field line \#1 all the way back to the inner electrode. Since the net axial flux observed at the probe array is $\sim 0$ (ignoring the small vertical vacuum field) the total plasma current leaving the gun $\left(\mathrm{I}_{\mathrm{p}}{ }^{\text {out }}\right)$ should match the total plasma current returning to the gun $\left(\mathrm{I}_{\mathrm{p}}{ }^{\mathrm{in}}\right)$ at the array height. Since these are not in fact equal, current must flow across field lines (i.e. $\# 1$ to \#2) somewhere below the position of the probe array to explain the imbalance. By definition, this is not a force-free current.

This is not the first reporting of cross-field current in supposedly force-free spheromaks. The SPHEX group reported that up $50 \%$ of the gun current went into the 
outer wall but only $10 \%$ of the gun flux diffused into it ${ }^{5}$. Measurements made on CTX showed similar results (Figures 16 and 17 of Ref. 14).

If the open field line volume forms rational or ergodic surfaces, then the cross field current observations can only be explained by current that is perpendicular to the surfaces and outward along the plasma minor radius. This would generate a toroidal $\mathrm{jxB}$ force on the plasma. If the open field line volume is stochastic, then it is impossible to predict the net direction of the cross-field current or any resulting force on the plasma. To determine the existence of a net $\mathrm{jxB}$ force and its direction we analyze the current flowing within the inner and outer electrodes that crosses a known magnetic field distribution (i.e. the vacuum field). If there is a torque on the plasma there must be an equal and opposite torque on the surrounding vessel. The following explains this analysis.

Consider our cylindrical coordinate system aligned with the geometric axis of the spheromak. The current-carrying solid bodies are the axisymmetric inner and outer electrodes. Inside the flux conserving copper, the magnetic field is the axisymmetric vacuum field. Current flowing within the copper may not be uniform or symmetric, but it is predominantly parallel to the wall, i.e. current perpendicular to surfaces is negligible. We can determine the total current $I$ flowing through an axisymmetric piece of a cylindrical shell. The force $F$ and associated torque $\tau$ about the axis are:

$$
\begin{aligned}
& d \vec{F}=I \vec{d} \times \vec{B} \\
& d \vec{\tau}=(I d \vec{l} \times \vec{B}) \times \vec{r} \\
& d \tau=I \cdot B_{\perp l} \cdot d l \cdot r \\
& d \tau=I \cdot\left(\frac{d \psi}{d \phi}\right) \\
& \tau=\frac{1}{\Delta \phi} \int_{\substack{\text { all flux } \\
\text { crossed }}} I d \psi
\end{aligned}
$$


Here, $d \vec{l}$ is the local current direction and $B_{\perp l}$ is the component of $\vec{B}$ perpendicular to the current. The differential flux $d \psi=B_{\perp l} d l r d \phi . \Delta \phi$ is the total azimuthal angle subtended by the material, which is simply $2 \pi$. The current flowing through the material is integrated over the total flux it crosses. We restrict our attention to the volume containing the plasma-facing coaxial electrodes.

Figure 9 shows the wall current calculated from the toroidal field at the walls using the rigid rotor analysis as a function of the flux it crosses in the copper, where the flux is defined as zero at the insulator and the maximum at the bottom centerline of the machine. The lower curve is produced from the toroidal field probes in the outer wall. The upper curve is the case in the inner electrode where the only toroidal field probe available is the one at the end of one insertable array. The area under each curve is proportional to the torque on that wall, and since these areas are unequal there is a net torque on the vessel and an equal and opposite torque on the plasma. Thus there must be a toroidal $\mathrm{jxB}$ force on the plasma in the counter-clockwise direction in the contour plots of Figure 4. This is opposite to the direction that the $n=1$ mode propagates (clockwise). We speculate that this $\mathrm{jxB}$ force is generated by a line-tying mechanism on the open field lines as a response to toroidal plasma rotation. Impurity ion velocity measurements have been very limited in SSPX and unavailable for this study, although a $60 \mathrm{~km} / \mathrm{s}$ toroidal ion flow in the same direction as the $n=1$ propagation was observed using an Ion Doppler Spectrometer in a different discharge configuration. ${ }^{15}$ Ion rotation in the same direction as the $n=1$ mode has also been confirmed in another spheromak ${ }^{16}$ and in a spherical torus ${ }^{8}$ that uses coaxial helicity injection. While the exact cause of this rotation is uncertain, any toroidal momentum on the open flux would cause a line-tying 
reaction. In the ideal limit, $\mathrm{E}=0$ in the copper, $\mathrm{E}_{\|}=0$ in the plasma, and therefore $\mathrm{E}_{\perp}=0=\mathbf{v} \mathbf{X} \mathbf{B}$ in the plasma on open flux. So in the ideal limit no motion perpendicular to the open field is allowed: cross field current is driven to exactly cancel it. For the more realistic case of finite resistivity $\eta$, we have $\eta \mathbf{j}=\mathbf{v x} \mathbf{B}$. The cross field current $j_{\perp}$ is just the amount necessary to hold $v_{\perp}$ constant through the action of a $j_{\perp} x B$ force on the plasma. The line tying current $j_{\perp}$ is the cross-field current we infer exists from the probe measurements.

\section{SUMMARY}

The coaxial gun used to sustain the SSPX spheromak has been shown to break many of the commonly held assumptions. The amount of gun flux utilized varies, even for identical machine settings, and this affects the helicity injection rate. When a large $\mathrm{n}=1$ mode exists during sustainment, the flux and current within the coaxial gun become asymmetric. The open field line plasma is not force free in this case, and we infer from measurements that a cross-field current exists. We speculate that this current is due to the line tying effect acting to counter plasma rotation. Measurements of the rotation profile are needed to quantify this work further.

\section{ACKNOWLEDGEMENTS}

We wish to thank Bob Geer and Richard Kemptner for diagnostic assembly and SSPX operation.

This work was performed under the auspices of the U.S. Department of Energy by University of California, Lawrence Livermore National Laboratory under contract W-7405-Eng-48. 


\section{Figure Captions}

FIG. 1. The SSPX cross section showing features referred to in the text.

FIG. 2. Representative time histories of the gun voltage and current (top) and three $B_{z}$ probes spanning the radius of the gun channel (bottom) during sustainment with an $n=1$ mode.

FIG. 3. Instantaneous magnetic field profiles at one toroidal angle in the gun channel for the shot shown in FIG. 2.

FIG. 4. Reconstruction of the $n=1$ mode structure in the gun channel from probe measurements and the application of a rigid-rotor model.

FIG 5. Axial flux stretched out of the gun channel versus time.

FIG. 6. Contours of $\lambda=\mu_{0} \mathrm{j}_{\mathrm{z}} / \mathrm{B}_{\mathrm{z}}$.

FIG. 7. Time history of net current flowing into and out of the gun within both plasma and the walls.

FIG. 8. Plot of wall current versus the vacuum flux it crosses to produce a torque. 


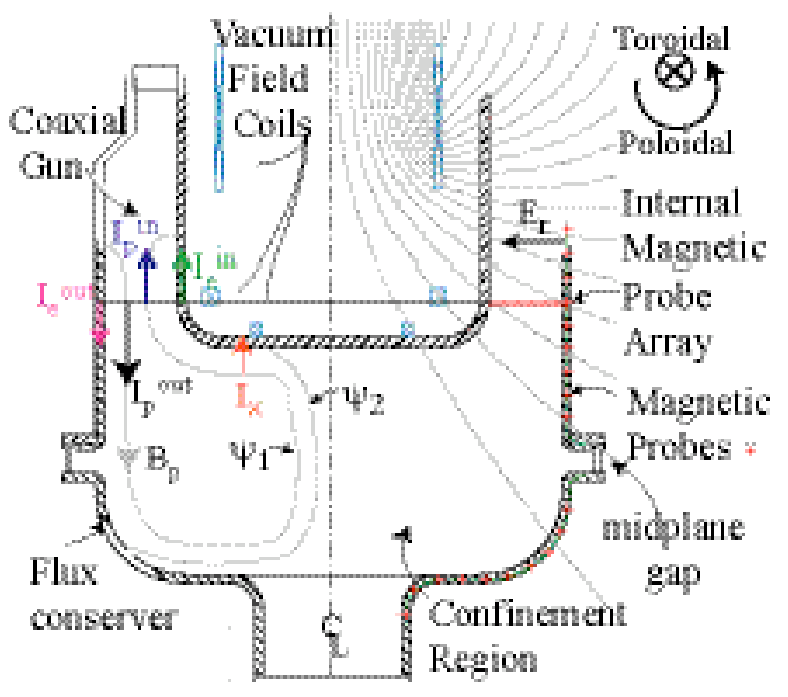

Figure 1 


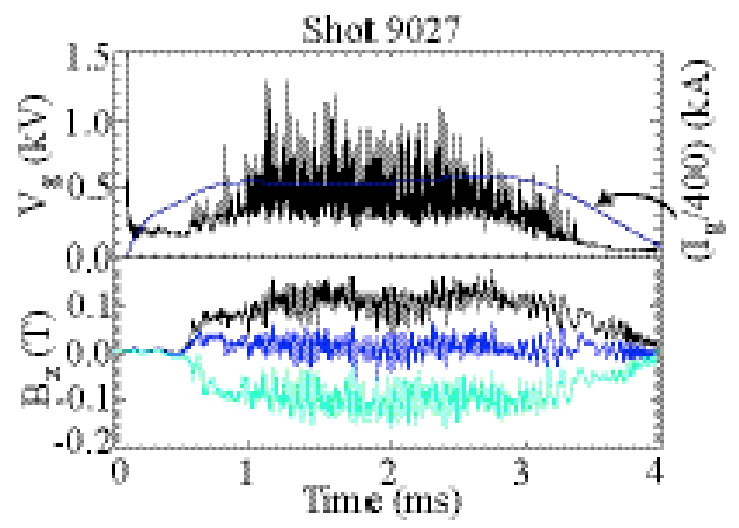

Figure 2 


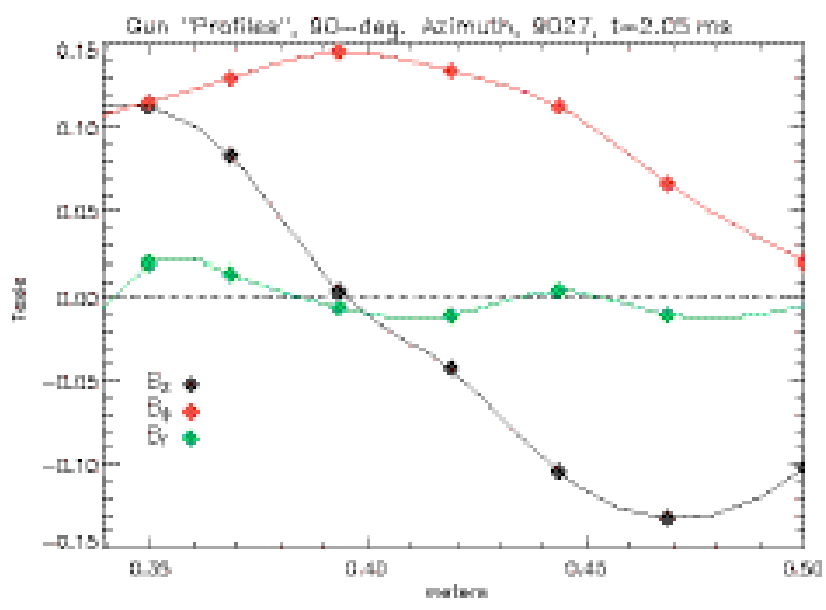

Figure 3 


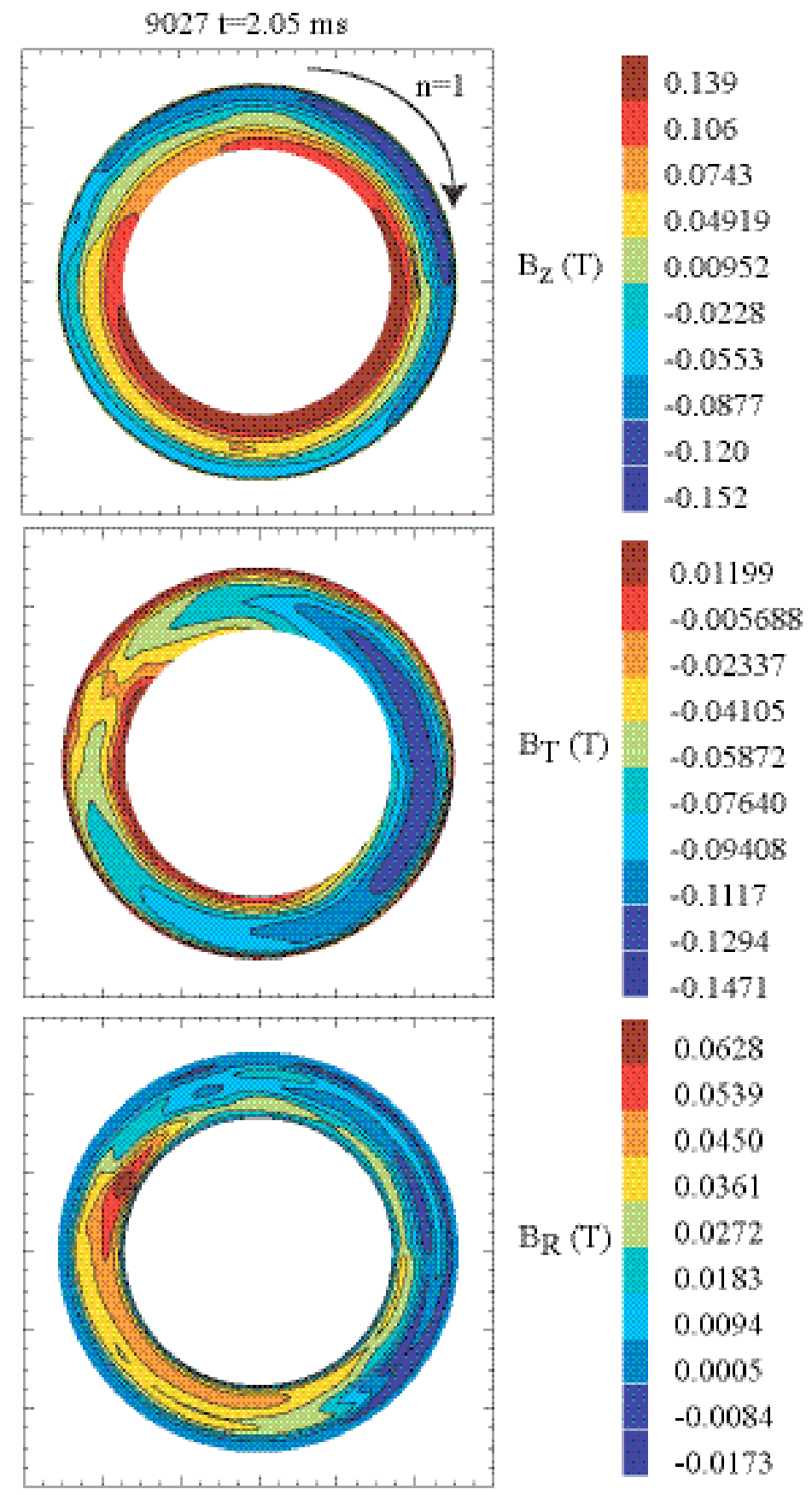

Figure 4 


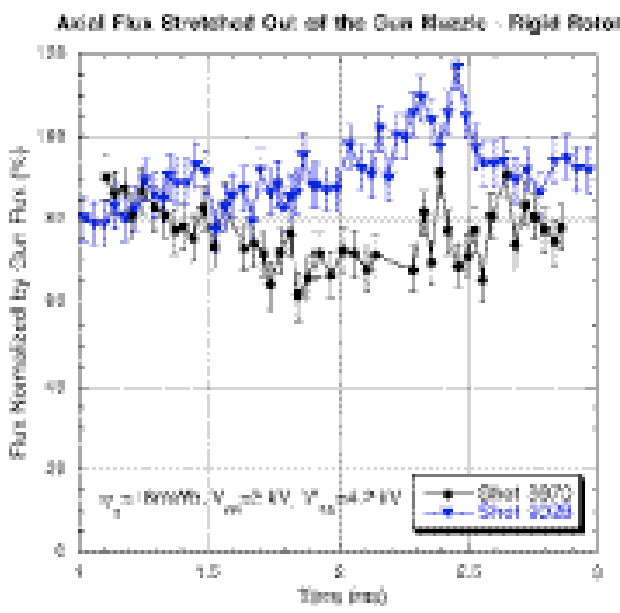

Figure 5 


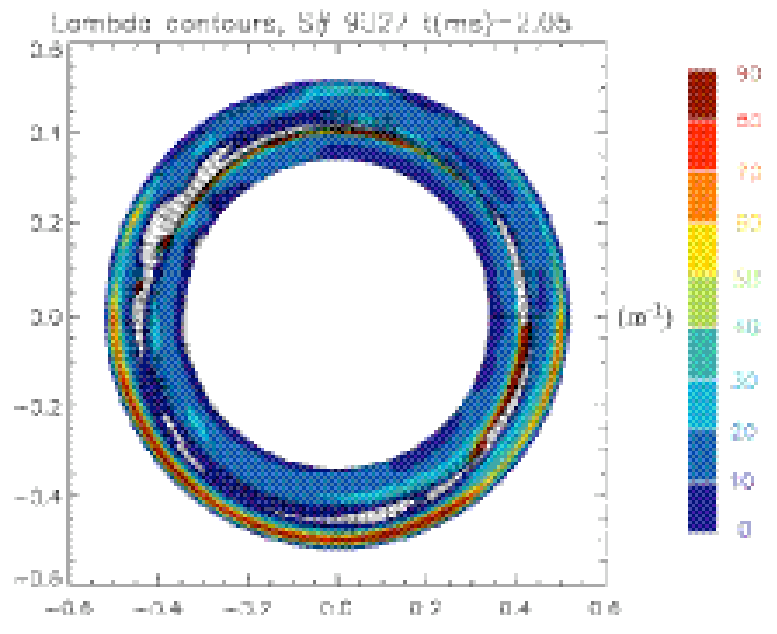

Figure 6 
Shot 9027 Canrent Distribution

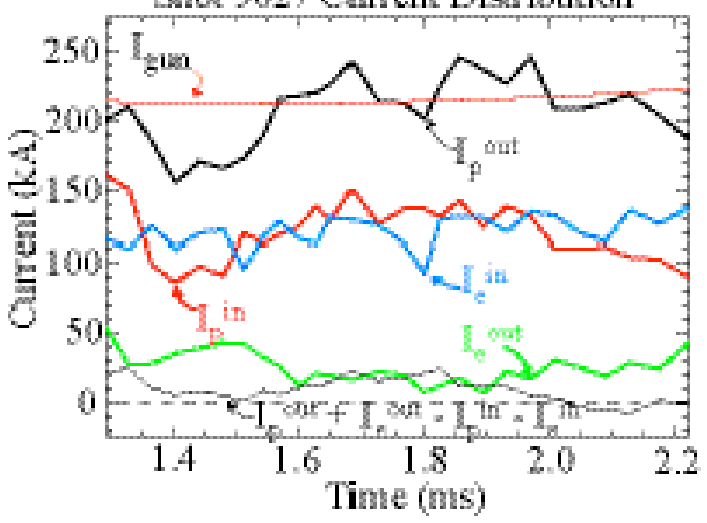

Figure 7 


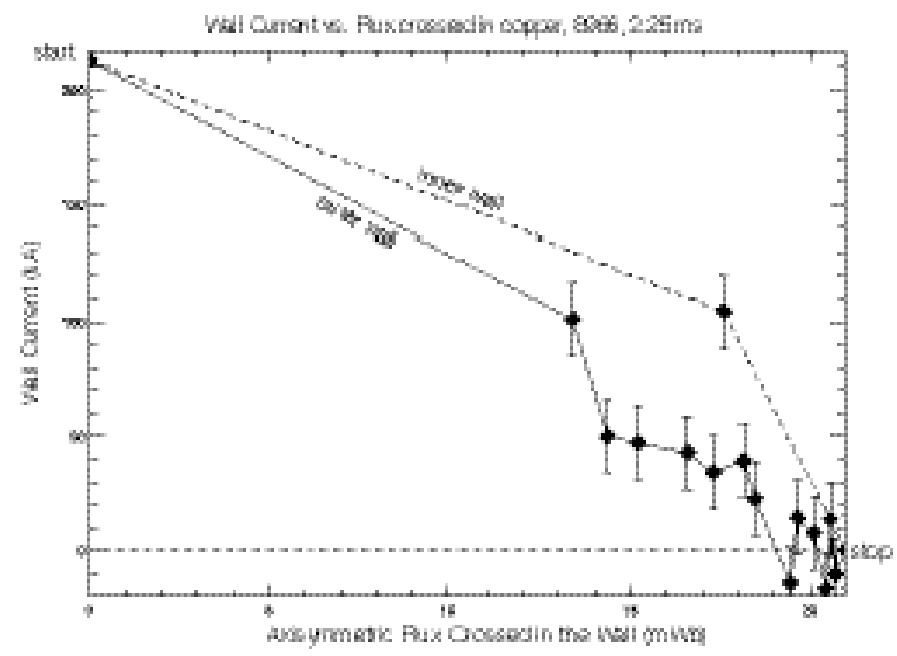

Figure 8 
${ }^{1}$ T.R.Jarboe, Plasma Phys. Controlled Fusion 36, 945 (1994).

2 J.B.Taylor, Phys. Rev. Lett. 33, 191139 (1974).

${ }^{3}$ C.W. Barnes et al., Phys. Fluids 29, 3415 (1986).

${ }^{4}$ A. al-Karkhy et al., Phys. Rev. Lett. 70, 1814 (1993).

${ }^{5}$ R.C. Duck et al., Plasma Phys. Controlled Fusion 39, 715 (1997).

${ }^{6}$ S. Woodruff and M. Nagata, Plasma Phys. Controlled Fusion 44, 1 (2002).

${ }^{7}$ C.R. Sovinec, J.M. Finn, and D. del-Castillo-Negrete, Phys. Plasmas 8, 2 (2001).

${ }^{8}$ K.J. McCollam and T.R. Jarboe, Plasma Phys. Controlled Fusion 44, 493 (2002).

${ }^{9}$ E.B. Hooper, L.D. Pearlstein, R.H. Bulmer, Nucl. Fusion 39, 863 (1999).

${ }^{10}$ S. Woodruff et al., Phys. Rev. Lett. 90, 095001 (2003).

${ }^{11}$ H.S. McLean et al., Phys. Rev. Lett. 88, 125004 (2002).

${ }^{12}$ H.S. McLean et al., Rev. Sci. Instrum. 72, 1 (2001).

${ }^{13}$ B.W. Stallard et al., Phys. Plasmas 10, 2912 (2003).

${ }^{14}$ J.C. Fernández et al., Nucl. Fusion 28, 9 (1988).

${ }^{15}$ M. Nagata, private communication.

${ }^{16}$ P.K. Browning et al., Phys. Rev. Lett. 68, 111718 (1992). 